\title{
Sondes optiques : innovations sur un capteur classique
}

par Alain CARTELLIER, du Laboratoire des Ecoulements Géophysiques et Industriels (LEGI) de Grenoble

Christian POUPOT, Eric CHAMBÉROD du Groupe d'Instrumentation et de Contrôle Electronique des Procédés (GICEP), Grenoble

Eric BARRAU, doctorant rattaché au LEGI et GICEP

\section{I 國 CONTEXTE DE L'ÉTUDE ET OBJEC- TIFS}

Parmi les capteurs disponibles, les sondes optiques constituent un outil essentiel pour l'investigation des écoulements gaz-liquide [1]. Sensibles à l'indice de réfraction de la phase entourant leur extrémité, elles délivrent en effet un signal proche de la fonction indicatrice de la phase gaz $X_{G}$, information clef pour ces écoulements. Le taux de vide $\alpha$ ou concentration locale de la phase gaz est alors obtenu par une sonde simple (mono-sonde). Des sondes doubles (bi-sondes) ou multiples donnent en outre accès à la vitesse et à la granulométrie de la phase gaz. Ces capteurs présentent le grand avantage de rester opérationnels dans pratiquement toutes les configurations d'écoulements gazJiquide. Enfin, leur simplicité d'emploi favorise leur utilisation en conditions industrielles, que ce soit pour de la mesure ou du contrôle.

\begin{abstract}
Depuis le premier prototype réalisé en 1969 , ces capteurs ont bénéficié des progrès techniques notamment en opto-électronique, et ils ont prouvé leur utilité dans de multiples études (voir article de synthèse [2]). Toutefois, leurs performances n'ont été évaluées que dans un nombre restreint de conditions d'écoulement [2], avec des sondes généralement difficiles à reproduire, et qui plus est, associées à des techniques de traitement de signal souvent ad hoc. Il n'est alors pas rare que l'utilisateur soit confronté à des formes de signaux non classiques, pour lesquels des difficultés d'interprétation apparaissent. D'autre part, il est souvent très difficile d'estimer l'incertitude entachant les mesures de taux de vide ou de vitesse. Ainsi, bien que ces capteurs soient devenus classiques, leurs performances restent mal connues. Les mêmes questions se posent d'ailleurs pour les sondes résistives (voir [2]).
\end{abstract}

Pour en améliorer la maîtrise, un critère objectif de comparaison entre sondes, fondé sur le concept de lon-

Optical probes are an essential component of the two-phase flow instrumentation, but their performances are still not well known, mainly due to a bad control of their sensitive tip, and to defects in signal processing. The DER-EDF, the LEGI and the GICEP have grouped together in order to improve the mastership of such probes. New sensitive tips geometries have allowed to simultaneously measure the gas phase characteristic function and its velocity with a single probe. A dedicated real time signal processing has been elaborated: its performances render it quite objective. The measuring ensemble has been checked in various air-water flows. The discrepancy with reference mesurements lies between 0 and $-13 \%$ for the void fraction as well as for the velocity, provided that the gas fraction is higher than $5 \%$. Beside, the real time processing has been extended to double-probes, but, in that case, a criteria remains which must be set by the user. Calibrations of bi-probes exhibit an error on velocity as high as $30 \%$. Thus, the measuring ensemble for single probes appears to be more reliable and accurate than the classical double-probe technique: it offers an elegant solution for the characterisation of gas/liquid flows. Various extensions are discussed. 
gueur de latence qui sera décrit au $\$ 2.1$, a été proposé au LEGI [3]. Il en a résulté une méthode originale de mesure de la vitesse d'interface à partir d'une mono-sonde, permettant ainsi d'accéder avec une sonde simple aux mêmes informations que celles issues de bi-sondes. Sa faisabilité a été démontrée dans le cadre d'un contrat entre la DEREDF et le LEGI [4], [5]. Cette opération de recherche a aussi permis de metre en ouvre une nouvelle procédure de traitement des signaux, bien moins sensible aux paramètres de traitement que les techniques classiques [2], mais au prix de temps de calcul prohibitifs.

Ces développements ont été poursuivis dans le cadre du GREDIC (GdR 1027), par une collaboration entre la DEREDF, le LEGI et le GICEP, permettant de couvrir l'ensemble des processus intervenant dans ces chaînes de mesure. Ce programme de recherche a vocation à réaliser et qualifier des systèmes temps réel, pour des mono-sondes avec mesure de la vitesse de la phase gaz aussi bien que pour des bi-sondes; il s'articule autour de trois types d'actions :

- mieux appréhender la physique de la détection des interfaces, qui combine réponse optique et forme instantanée de l'interface que contrôle l'hydrodynamique à l'échelle de la tête de sonde. En effet, l'établissement d'un traitement fiable nécessite de savoir relier le signal détecté à la position relative de la sonde par rapport à l'interface. Ce type d'information est d'autant plus difficile à établir que les extrémités de sondes et donc l'allure des signaux bruts présentent une grande variabilité. Il faut donc optimiser et maitriser la géométrie des têtes de sondes, avant d'en analyser la réponse lors du perçage d'interfaces élémentaires:

- réaliser un traitement de signal, fondé sur la connaissance acquise sur les processus à petite échelle, et permettant d'extraire les informations brutes pertinentes en temps réel. Dans une phase de post-traitement, ces données doivent être transformées en grandeurs physiques intervenant dans les modélisations :

- qualifier les chaines de mesure résultantes sur des écoulements représentatifs afin de mettre en évidence l'erreur due au caractère intrusif de cette technique, aspect qui peut conduire à l'évitement de la sonde par les bulles ou à des modifications de leur vitesse. Soulignons qu'à faible vitesse, l'organisation des phases peut aussi être perturbée dans le voisinage du capteur en raison de la présence du support. La multiplicité des variables intervenant dans ces processus rend une analyse systématique difficilement envisageable. Nous avons choisi de faire porter la campagne de qualification sur quelques écoulements bien contrôlés, en espérant pouvoir ainsi dégager des tendances sur l'évolution de l'incertitude avec par exemple la taille des inclusions gazeuses ou encore la vitesse de la phase porteuse.

Le présent article dresse un bilan des résultats obtenus dans le cadre de cette collaboration. Après un rappel des phénomènes intervenant lors du perçage d'interfaces (\$2.1), les nouvelles têtes de mono-sondes seront présentées au $\$ 2.2$ ainsi que leurs réponses lors de la traversée d'interfaces élémentaires. L'architecture et les performances du système de traitement associé seront décrites au $\$ 2.3$ et la qualification des chaînes de mesures complètes pour mono-sondes fera l'objet du $\$ 2.4$. Le cas des bisondes sera brièvement évoqué au $\$ 3$.

\section{DONO-SONDES}

\subsection{Perçage d'interfaces contrôlées et longueur de la- tence}

A l'échelle de la tête de sonde, les processus optiques dépendent de l'indice de réfraction de la phase liquide. mais aussi de la forme de l'extrémité, de sa structure, de son état de surface et, pour les capteurs à fibres optiques, des modes de propagation de la lumière. A cette même échelle, l'hydrodynamique du perçage, quant à elle, recouvre trois étapes: la déformation de l'interface avant impact, la dynamique du drainage précédant la rupture et conduisant à l'apparition d'une ligne triple, et enfin la dynamique de l'assèchement ou du mouillage assurant la fin de la transition entre phases (fig. l). L'extrémité sensible intervient ici par sa forme, son état de surface mais aussi sa nature (via l'attraction exercée par le solide).

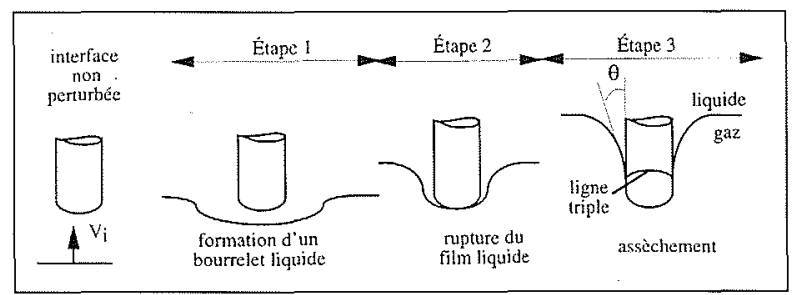

1. Evolution schématique de l'interface lors d'un perçage perpendiculaire.

La complexité de ces mécanismes nous a incité à examiner dans un premier temps l'allure des signaux résultant du passage de sondes au travers d'interfaces air-eau contrôlées en forme et vitesse. Quelle que soit la sonde, les durées de transition les plus longues correspondent au perçage dans le sens liquide $\rightarrow$ gaz, en raison de la plus forte inertie de la phase liquide. Il a été montré que, pour des interfaces planes, la durée $T m$ de la transition $L \rightarrow G$ est fortement corrélée à la vitesse d'interface $V i$. Le tracé de la longueur de latence $L$, définie comme le produit $T m V i$, en fonction de $V i$ (fig. 2), fait apparaître des caractéristiques fondamentales du perçage. Au-delà d'une vitesse minimale $V c$, $L$ reste pratiquement constante $\left(=L^{*}\right)$, indiquant que $T \mathrm{~m}$

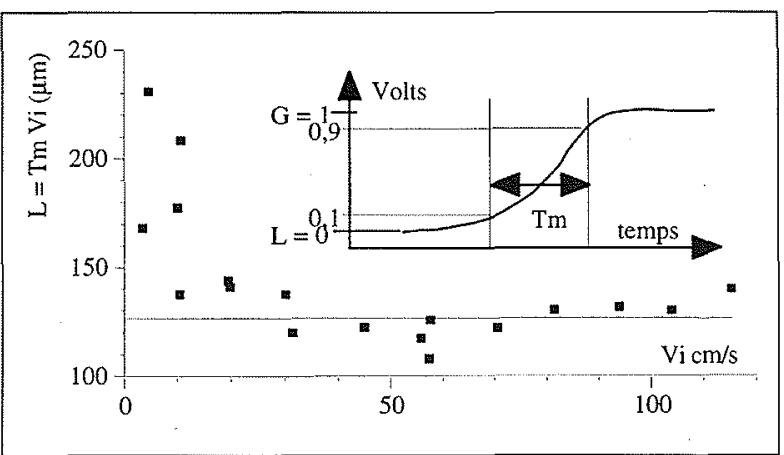

2. Longueur de latence pour une sonde monofibre étirée ([3]). 
s'identifie à un temps de transit à la vitesse de l'interface et sur une distance sensible $L^{*}$. A faible vitesse, cette réponse cinématique est altérée par la dynamique propre de la ligne triple, ce qui accroît la durée des transitions. Cette résolution spatiale $L^{*}$ permet une comparaison objective entre sondes [3].

A partir de ces résultats, il est donc possible, connaissant $T m$, de déduire la vitesse d'interface. Deux difficultés apparaissent lorsque l'on veut exploiter ce principe sur une sonde implantée au sein d'un écoulement diphasique. D'une part, les interactions sonde/interfaces mettent en jeu d'autres variables comme la courbure ou l'angle d'impact, variables qui peuvent affecter la corrélation établie en interface plane. D'autre part, les mesures se devant d'être compatibles avec les concepts en usage dans les modèles, il est souhaitable d'accéder à une vitesse phasique moyenne de la phase gaz. Pour cela, choix a été fait de corréler $T m$ au module $V o$ de la vitesse du centre de masse de chaque inclusion gazeuse. En géométrie plane, on s'attend donc à ce que $T m$ s'exprime par la fonctionnelle suivante :

$$
T m=f\left(V_{o}, \beta, \gamma, R\right)
$$

où $R$ désigne le rayon de courbure de l'interface, $\beta$ l'angle entre l'axe de la sonde et la normale à l'interface au point d'impact, et y l'angle entre l'axe de la sonde et la direction de la vitesse d'ensemble de l'inclusion gazeuse (fig. 3).

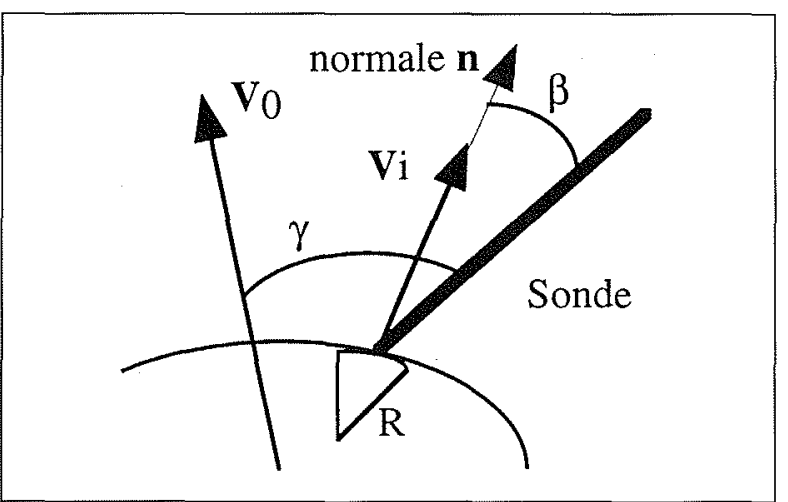

3. Paramètres d'interaction sonde-interface en géométrie plane.

Plusieurs installations expérimentales ont été réalisées pour étudier la fonction (1). Il est apparu que le rayon de courbure n'intervient pas tant qu'il reste grand devant la taille de la sonde (de l'ordre de 40 fois). En revanche, l'angle d'impact $\beta$ affecte très fortement la durée des transitions dès qu'il excède une dizaine de degrés. Cette difficulté oblige, lors du traitement, à sélectionner les transitions significatives pour la mesure de vitesse. Ces aspects sont détaillés dans [4]. La question de l'influence de l'angle $\gamma$ restait toutefois ouverte.

Par ailleurs, l'allure des transitions ainsi que leur durée varient selon la sonde considérée. Ainsi, la plupart des signaux obtenus lors de perçages $L \rightarrow G$ à incidence normale $\left(\beta=0^{\circ}\right)$, présentent un pic au démarrage de la transition. Ce comportement n'est cependant pas systématique, et semble dépendre de l'état d'encrassement de la tête de sonde. Il en est de même pour la présence ou l'absence d'un «overshoot » en fin de transition $L \rightarrow G$, ou au début de la transition $G \rightarrow L$, ainsi que pour la stabilité ou la décroissance du signal au niveau gaz. Cette diversité est liée en grande partie au manque de reproductibilité des têtes de sondes que ce soit sur la forme ou sur les dimensions. Notons que ces résultats ont été obtenus avec des sondes disponibles dans le commerce et fabriquées par étirage à chaud (Photonetics, RBI). Pour progresser dans l'analyse des interactions sonde/interface, il devenait impératif de mieux contrôler les extrémités sensibles, ce qui nous a conduit à concevoir de nouvelles sondes.

\subsection{Optimisation des têtes de sondes}

\subsubsection{Définition et réalisation de nouvelles géométries}

Le problème consistait à définir des géométries qui, tout en assurant la détection des poches de gaz avec un signal de bonne dynamique, permettent un contrôle à priori de la longueur de latence. D'une part, il paraissait utile de tester à nouveau des sondes à extrémité conique (ou $1 \mathrm{C}$ ), proposées par Abuaf et al. [6]. D'autre part, à partir du savoir faire acquis sur les sondes du commerce, il nous semblait qu'une portion cylindrique sur l'extrémité sensible pouvait être identifiée à la longueur de latence, et répondre ainsi à nos exigences. Nous avons donc considéré des formes cylindro-conique (ou 2C) et conique-cylindro-coniques (ou $3 \mathrm{C}$ ).

L'analyse du comportement optique de ces formes type a été menée sur la base d'une simulation en optique géométrique. La difficulté provenant du caractère multimodal des fibres employées, la lumière incidente a été idéalisée par des sources identiques, distribuées de façon homogène dans une section droite, et émettant dans un angle solide limité par l'ouverture numérique de la fibre. L'interface traversée par la sonde a été représentée par un saut d'indice selon un plan perpendiculaire à l'axe de la fibre. Seul le couple eau-air a été analysé.

Pour des cônes parfaits, la simulation prédit la faisabilité de la détection de phase jusqu'à des angles au sommet de $30^{\circ}$, limite bien inférieure à celle calculée par Abuaf et al. La longueur de latence ne peut être aisément corrélée à la géométrie, excepté pour un demi-angle au sommet de $45^{\circ}$. Par ailleurs, la fabrication ne permettant pas d'aboutir à des cônes parfaits, des cônes tronqués en extrémité ont été simulés. Ce clivage d'extrémité accroît la dynamique des signaux ce qui explique le bon fonctionnement de sondes effilées (angle au sommet de quelques degrés). La transition est d'autant plus rapide que la dimension de la partie clivée croît et/ou que l'angle au sommet est fort : une telle sensibilité de la longueur de latence à la géométrie est a priori difficilement compatible avec un bon contrôle de fabrication. On se référera donc aux tests expérimentaux pour jauger l'aptitude des sondes coniques à mesurer des vitesses.

Les signaux simulés pour une sonde $2 \mathrm{C}$, illustrés figure 4 , sont constitués d'une première transition suivie d'un plateau (lorsque l'interface glisse sur la partie cylindrique), et se terminant par une deuxième transition (lorsque l'interface glisse sur le cône arrière). Pour obtenir un plateau à une amplitude médiane entre les niveaux liquide et gaz, le diamètre de la partie cylindrique est crucial. La 
simulation est ici fort utile car ce choix dépend de l'ensemble de la géométrie de la tête. La durée du plateau intermédiaire crô̂t linéairement avec la longueur du cylindre : ces formes permettent donc un contrôle a priori de la longueur active. Les sondes $2 \mathrm{C}$, en raison de leur extrémité clivée, détectent l'interface avant qu'elle ne touche la sonde, ce qui se traduit par un pic marqué au démarrage du signal (voir fig. 8). Cette détection de proximité devient négligeable dans le cas de sondes $3 \mathrm{C}$ du fait de la finesse de leur tête: ce type de sonde s'avère donc encore mieux adapté à la mesure de la vitesse d'interfaces.

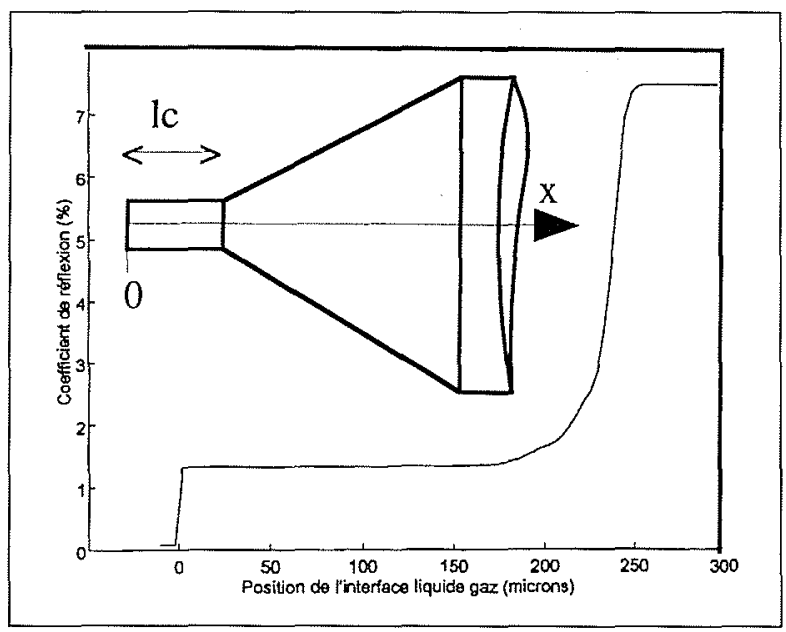

4. Transition $L \rightarrow G$ simulée pour une sonde $2 C$ ( $l c=\mathbf{2 0 0} \boldsymbol{\mu m}$, sans détection de proximité).

Ces géométries ont été réalisées à partir de fibres optiques en silice de diamètre extérieur $140 \mu \mathrm{m}$. La technique originale mise en oeuvre, différente d'un étirage à chaud, fait l'objet d'un brevet. Elle permet de reproduire les formes et les dimensions, et assure une bonne qualité de l'état de surface (fig. 5).

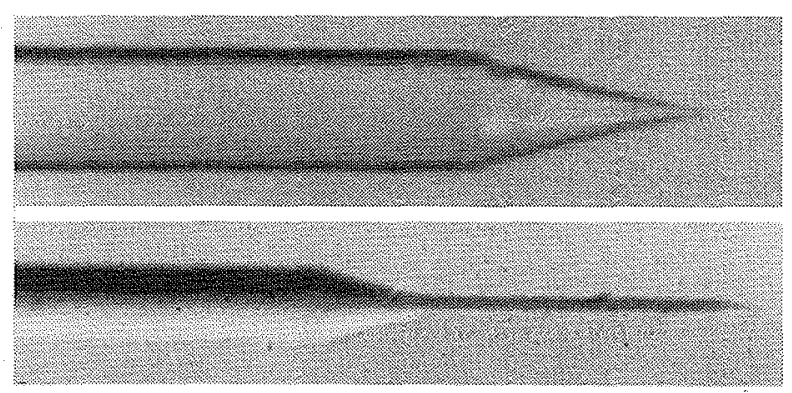

5. Exemples de têtes de sondes.

\subsubsection{Réponse expérimentale des sondes $1 \mathrm{C}$}

Plusieurs sondes coniques d'angle au sommet de $30^{\circ}$ ont été réalisées afin de tester la reproductibilité de leur réponse. En premier lieu, les écarts sur la dynamique des signaux peuvent atteindre un facteur 3 . Ces différences, liées à la qualité de la pointe, sont bien prédites par les simulations pour des cônes tronqués. Les longueurs de latence, obtenues par perçage de bouchons isolés, sont présentées figure 6 (pour des seuils situés à 10 et $80 \%$ de l'écart entre niveaux). Pour chaque sonde, $\mathrm{L}$ est reproductible à mieux que $\pm 5 \%$, excepté à faible vitesse c'està-dire dans la zone influencée par la dynamique de la ligne triple. De plus, l'ordre de grandeur de $L$ est bien conservé entre les sondes EB08 et EB10 qui sont géométriquement très semblables. En revanche, la sonde EB09 a un comportement singulier, probablement lié à un défaut géométrique prononcé situé à la base du cône. L'ordre de grandeur de la longueur de latence expérimentale ( $\approx 70 \mu \mathrm{m}$ à $50 \mathrm{~cm} / \mathrm{s})$ s'avère correspondre aux résultats de la simulation pour un cône parfait, alors que la dynamique est en accord avec la simulation d'un cône tronqué. Cette contradiction disparaît si l'on invoque le fait que, pour une sonde conique, la durée de la transition est sensible à la présence d'un film liquide $[2,6]$ : l'interface plane utilisée dans les simulations constitue ici une idéalisation abusive.

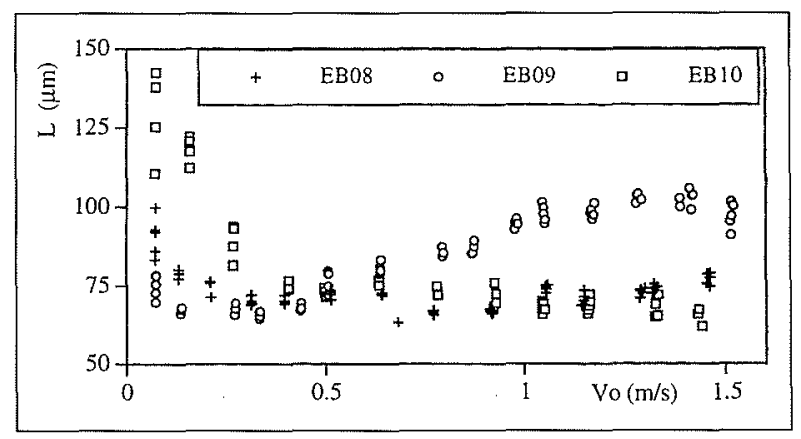

6. Evolution de la longueur de latence pour trois sondes de type $1 \mathrm{C}$.

L'influence des angles $\gamma$ et $\beta$ a été analysée sur une nouvelle installation où des bulles isolées sont produites dans un jet liquide vertical à débit variable, et où la sonde peut être inclinée entre 0 et $90^{\circ}$ par rapport à la verticale. La vitesse de la bulle avant impact, sa taille ainsi que les angles $\gamma$ et $\beta$, sont déduits de clichés tels que ceux présentés figure 7 , obtenus au travers d'un microscope. Les dernières positions de la bulle permettent d'estimer son ralentissement, et donnent une idée de l'ampleur de la déformation de l'interface

Les essais ont été conduits à vitesse de bulle fixée $\left(V_{O} \approx 56 \mathrm{~cm} / \mathrm{s}\right)$, d'une part en perçage perpendiculaire $\left(\gamma=0^{\circ}, \beta=0^{\circ}\right)$ et d'autre part pour une sonde inclinée à $\gamma=30^{\circ}$ impactant le nez de la bulle $\left(\beta=30^{\circ}\right)$. Les durées $T m$ obtenues se sont avérées identiques à $10 \%$ près, incertitude qui est du même ordre que le ralentissement de la bulle. L'influence de l'angle $\gamma$ semble donc négligeable. En revanche, quelques données obtenues pour une sonde impactant loin du nez de la bulle ont confirmé la forte influence de l'angle $\beta$. L'analyse de l'évolution de $L$ avec $\gamma$ et $\beta$ n'a pas été systématique car une difficulté supplémentaire, liée à l'état de surface de la sonde, est apparue. En effet, pour une sonde polluée par des particules (en l'occurrence des particules de rouille), le démarrage de la transition est retardé par rapport à la même sonde nettoyée. De plus, les signaux délivrés par une sonde encrassée ne présentent pas «d'overshoot» en fin de transition, et la 

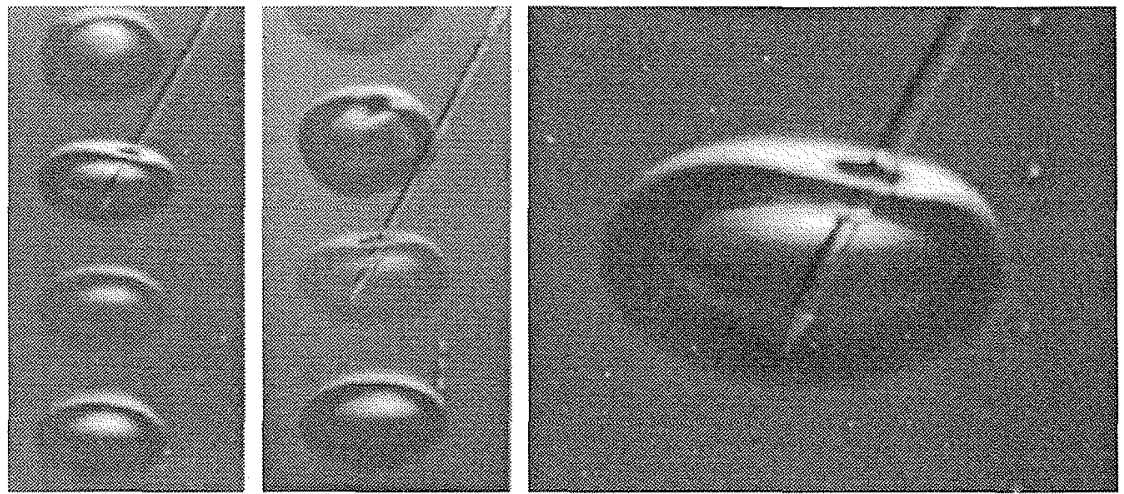

7. Clichés permettant la mesure de l'influence de l'angle entre sonde et vitesse.

durée $T m$ est deux fois plus courte que pour une sonde réputée propre. Ces comportements sont dus à l'inhibition du drainage induit par les impuretés attachées à la tête de sonde et à la persistance d'un film liquide. Il est clair que ces phénomènes, non contrôlables, peuvent conduire à de sérieuses difficultés de mesure selon les conditions opératoires.

\subsubsection{Réponse expérimentale des sondes $2 \mathrm{C}$ et $3 \mathrm{C}$}

Pour les sondes $2 \mathrm{C}$ et $3 \mathrm{C}$, la longueur le de la partie cylindrique (voir fig. 4) a été fixée à environ un millimètre afin de tester aisément ces nouvelles formes. Le diamètre du cylindre choisi conduit à une amplitude médiane pour le plateau. Là encore, la dynamique fluctue entre sondes (dans un rapport 3 ), toujours en raison de l'influence de la pointe. Comme prévu par la simulation, les transitions présentent des paliers (fig. 8) ainsi qu'une détection de proximité bien marquée pour les sondes $2 \mathrm{C}$ : ce demier phénomène disparaît avec les sondes $3 \mathrm{C}$ dont la transition monotone est mieux adaptée à l'automatisation du traitement. Les mesures de longueur de latence confirment que la transition correspond au temps de transit de l'interface le long de la partie cylindrique, modulo une contribution du cône de base de l'ordre de $10 \%$ de $L$ (fig. 9). On note aussi la bonne reproductibilité $(\approx 5 \%)$ à la fois pour une sonde donnée et entre sondes. Enfin, pour des sondes $3 \mathrm{C}$, la longueur de latence ne varie pratiquement pas $(<5 \%)$ lorsque l'angle d'impact $\beta$ évolue entre 0 et $40^{\circ}$, plage bien supérieure à celle obtenue pour des sondes étirées (cf. $\$ 2.1 .1$ ). Bien que l'influence de l'angle $\gamma$ doive encore être testée, les sondes $3 \mathrm{C}$ constituent d'ores et déjà un très bon outil pour simultanément accéder à la fonction indicatrice de phase et à la vitesse de la phase dispersée.

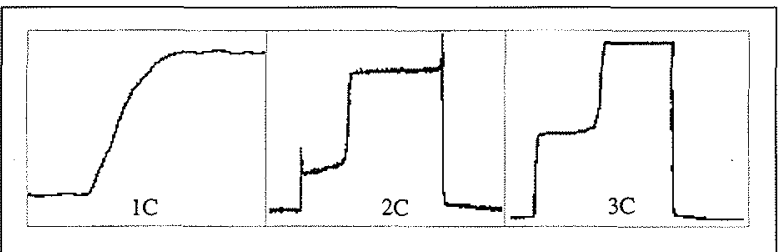

8. Signaux délivrés par des sondes 1C (transition $L \rightarrow G$ seule), $2 \mathrm{C}$ et $3 \mathrm{C}$.
Remarquons enfin, qu'au-delà de la vitesse critique, la longueur de latence est sensiblement croissante avec la vitesse de l'interface.

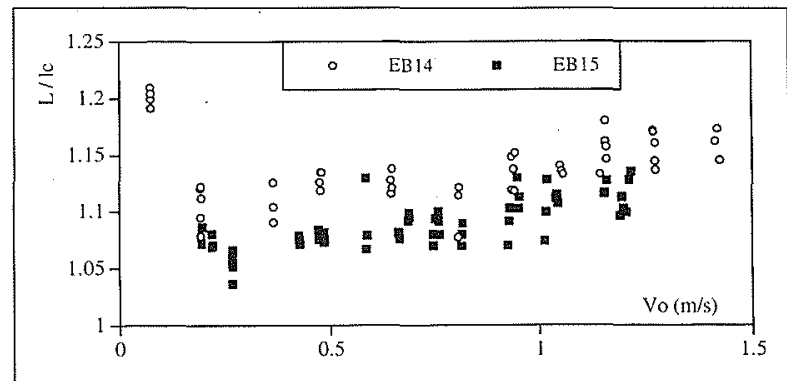

9. Rapport $L / l c$ pour deux sondes de type 3C.

\subsection{Traitement de signal temps réel}

\subsubsection{Objectifs du traitement et architecture du système}

L'exploitation des signaux bruts doit fournir la fonction indicatrice de phase ainsi que les durées des transitions $L \rightarrow G$ significatives. Ceci revient à identifier les instants d'arrivée $T A i$ (événement $A$ ) et de départ $T B i$ (événement $B$ ), ainsi que les dates de démarrage $T C i$ (événement $C$ ) et de fin $T D i$ (événement $D$ ) des transitions pour chacune des bulles $(i=1, \ldots, N)$ perçues par la sonde.

Il n'était pas possible de reprendre telles quelles les procédures mises en œuvre dans le traitement en différé [4], les opérations de filtrage et de lissage utilisées étant trop lourdes pour un processeur temps réel. Un nouvel organigramme de détection a donc été imaginé, qui intègre les acquis sur les interactions sonde/interface, et qui reste compatible avec les capacités de stockage et les vitesses de traitement du processeur. Cet organigramme est fondé sur une procédure de reconnaissance de formes pour laquelle un brevet est déposé. Nous restreindrons donc la description du système à son architecture générale.

Le signal brut issu de circuits de détection du commerce, et calé entre 0 et 5 Volts, est digitalisé sur 10 bits (après filtrage), la fréquence de conversion fe pouvant atteindre $1 \mathrm{MHz}$ qui est la valeur maximale autorisée par l'étage de 
détection. Les données sont transférées à un processeur de signal (DSP modèle 56001 de Motorala à virgule fixe), dont la puissance de calcul est de 10 Mips. Larchitecture est complétée par une mémoire externe de stockage (SRAM de 128 Kmots de 24 bits), une horloge assurant la datation absolue des événements, une mémoire FIFO permettant une communication unidirectionnelle vers un superviseur $(P C)$, et une liaison RS232 avec le $P C$. Ce système délivre en temps réel les dates caractéristiques et les amplitudes atteintes pour chaque inclusion gazeuse. En cours de mesure, le $P C$ affiche les statistiques sur les durées de transition $\mathrm{Tm}$ et sur les temps de présence dans la phase $\operatorname{gaz} T G(=T B-T A)$. Les capacités minimales du système permettent de détecter 5120 inclusions gazeuses avec mesure de la vitesse, ou 11600 inclusions pour la seule mesure de $X_{O}$. La durée d'analyse maximale est de $35 \mathrm{~min}$ pour $f e=1 \mathrm{MHz}$. A titre d'exemple, le système a été capable de suivre une cadence de 10000 bulles par seconde (mesure de a seul, $f e=500 \mathrm{KHz}$ ), ainsi que de caractériser des inclusions sub-millimétriques à une vitesse de $9 \mathrm{~m} / \mathrm{s}$

\subsubsection{Traitement de l'information en différé}

Pour l'étape de post-traitement, les données brutes, constituées par la suite des dates $T A i, T B i, T C i$ et $T D i$ $(i=1 \ldots N)$ sont transmises au $P C$. Fonction indicatrice du gaz $X_{G}$ et taux de vide sont déduits des TAi et TBi. Les vitesses d'ensemble Voi des inclusions sont calculées en inversant la corrélation $T m=f\left(V_{0}\right)$ adaptée à la sonde et aux seuils $C$ et $D$ utilisés. La vitesse moyenne de la phase gaz notée $V g$, est estimée en pondérant chaque valeur discrète de vitesse Voi par le temps de présence dans la phase gaz TGi correspondant soit:

$$
V_{g}=\left[\sum_{i \in[1, N]} T G i V_{o i}\right] / \sum_{i \in[1, N]} T G i
$$

Les vitesses n'étant mesurées que sur un sous-ensemble des $N$ événements détectés, il est possible d'attribuer une vitesse aux événements qui en sont dépourvus par simple interpolation temporelle. Une nouvelle vitesse moyenne de gaz est alors définie sur les $N$ événements. Les cordes intersectées par la sonde sont données par les produits $T G i$ Voi, ce qui permet d'accéder à la distribution croisée corde-vitesse: cette représentation facilite l'identification des populations d'inclusions présentes dans l'écoulement (cas des écoulements à bouchons par exemple). D'autres analyses sont possibles : évolution temporelle du taux de vide, test de convergence, étude de transitoires, détection de poches de gaz très peu probables etc... Par ailleurs, la distribution de tailles est reconstruite au moyen de la procédure de Clark et Turton [7]. Une telle opération requiert nombre d'hypothèses restrictives sur la dynamique de la phase dispersée (voir [2]) et nécessite de figer la forme des inclusions. Ces limites en réduisent l'intérêt pratique, d'autant plus que le résultat est très sensible à la présence de discontinuités sur l'histogramme de cordes. Enfin, la densité daire interfaciale, essentielle pour les processus de coalescence et de morcellement, est calculée. Là encore, il s'agit d'une estimation compte tenu des hypothèses nécessaires à la conduite du calcul [2].

\subsubsection{Critères de détection et optimisation}

Avant d'évoquer les critères, résumons les acquis sur l'interprétation des signaux.
Pour des sondes non contaminées, à pointe effillée (sondes étirées) ou conique, les tests de perçage montrent que le démarrage de la transition correspond à larrivée de l'interface sur l'extrémité du capteur. Il s'agit ici de l'interface déformée. Pour remonter à la position de l'interface non déformée, il faudrait conduire une analyse de l'hydrodynamique permettant de corriger la détection réalisée par la sonde. Notre but étant de mettre en oeuvre un traitement opérationnel, cette étape a été ignorée (d'autant plus que les premiers résultats obtenus sur ce problème montrent que la déformation est de l'ordre de 15-20 $\mu \mathrm{m}$ pour les sondes considérées ici [8]). Par ailleurs, il est illusoire de vouloir détecter des événements aussi faibles que le bruit présent sur le signal (il est préférable d'améliorer la dynamique en optimisant le capteur). Le critère retenu pour l'apparition d'un événement est donc fondé sur une tension supérieure au niveau liquide plus bruit. La connaissance précise du niveau liquide $V L$, et de l'amplitude du bruit crête à crête $V B$ (autour de $V L$ ) est donc essentielle.

Pour lensemble des sondes, la transition dans le sens $G \rightarrow L$ est brutale puisque le signal chute de 80 a $90 \%$ de sa valeur pour un déplacement de l'interface de l'ordre de $10 \mu \mathrm{m}$. Des comportements similaires avaient déjà été mis en évidence pour les sondes étirées [4]. La détection de la fin d'une bulle est donc fondée sur l'occurrence de tensions voisines du niveau liquide. La valeur du palier gaz. déterminée pour chaque événement, permet de remonter au début de la transition gaz $\rightarrow$ liquide par l'intermédiaire d'un critère de niveau (CS3). Ainsi, la connaissance préalable du niveau gaz $V G$ n'est pas nécessaire à l'identification des instants début et fin de bulle. Il en résulte que, contrairement aux procédures de seuillage courantes [3], le système comptabilise tous les événements vus par la sonde.

Les durées des transitions $L \rightarrow G$ ne sont mesurées que pour les signaux correspondant à une transition complète entre liquide et gaz, c'est-à-dire ceux pour lesquels le palier gaz est bien marqué. Les signaux atypiques, comme ceux produits lors d'un impact tangentiel sur une interface, ne domnent pas lieu à une mesure de vitesse. Les critères de sélection introduits à ce stade sont similaires à ceux employés dans le traitement en différé [4]. Notons que la priorité est donnée à la détection de la fonction indicatrice de phase, ce qui, en cas de surcharge du processeur, peut conduire à ne plus mesurer la durée des transitions jusqu’à ce que le retard soit comblé.

Sans détailler les organigrammes, signalons que sept paramètres contrôlent le traitement. Le tableau / illustre la faible interdépendance des critères retenus. Le coefficient de sécurité sur le bruit CS1 n’intervient que de façon conditionnelle, $A$ étant essentiellement fonction de la précision de l'évaluation du bruit. CS2 et $A P$ permettent de trier les signaux exploités en vitesse. Pour une phase porteuse d'eau ou de fréon. les amplitudes des paliers gaz restent stables pour des perçages complets: l'amplitude minimale $A P$ ne joue alors aucun rôle. Les seuils $C$ et $D$ sont fixés par le choix de la corrélation temps de montée/ vitesse ( $T m$ est le plus souvent défini pour les seuils 10 et $90 \%$ ). En raison de la raideur des fronts de descente, l'effet de CS3 est quasiment neutre. Les deux paramètres les plus critiques sont donc $A F$, qui contrôle la reconnaissance de forme et affecte la détection des événements $B, C$ et $D$, et $C S 2$. L'optimum pour $A F$ résulte de contraintes sur la précision de la mesure et sur la durée de traitement d'un événement. Elle s'explicite en fonction de la dynamique 
Tableau 1. - Influence des paramètres de traitement sur chaque date caractéristique (influence directe $=X$, conditionnelle $=\star$, sans effet $=0$ ).

\begin{tabular}{|l|c|c|c|c|c|c|c|}
\hline $\begin{array}{l}\text { Critère } \Rightarrow \\
\text { Date }\end{array}$ & $\begin{array}{c}\text { Sécurité } \\
\text { CS1 }\end{array}$ & $\begin{array}{c}\text { Analyse de } \\
\text { forme AF }\end{array}$ & $\begin{array}{c}\text { Durée } \\
\text { palier CS2 }\end{array}$ & $\begin{array}{c}\text { Amplitude } \\
\text { palier AP }\end{array}$ & $\begin{array}{c}\text { Seuil } \\
\text { bas C }\end{array}$ & $\begin{array}{c}\text { Seuil } \\
\text { haut D }\end{array}$ & $\begin{array}{c}\text { Fin de } \\
\text { bulle CS3 }\end{array}$ \\
\hline TA & $\bullet$ & 0 & 0 & 0 & 0 & 0 & 0 \\
TB & 0 & $\times$ & 0 & 0 & 0 & 0 & $X$ \\
TC & 0 & $X$ & $X$ & $\times$ & 0 & 0 \\
TD & 0 & $X$ & - & 0 & $X$ & 0 \\
\hline
\end{tabular}

des signaux et du rapport signal sur bruit $(R S B)$, ce qui permet au système de calculer sa valeur dés lors que les niveaux $V L, V B$ et $V G$ sont connus. Quant à $C S 2$, la valeur recommandée évolue entre 2 et 5 .

\subsubsection{Sensibilité aux réglages et aux critères, reproductibi- lité}

Le système a été pourvu d'une phase d'apprentissage permettant la détermination automatique des niveaux de référence $V L, V B$ et $V G$. Cette procédure ne dispense pas l'opérateur de régler le signal analogique issu du capteur. Une analyse expérimentale de sensibilité a donc été conduite sur la variable la plus critique à savoir $V L$. Pour cela, la valeur du niveau liquide déclarée au système (VLo) est figée, et c'est la tension VLeff en sortie de capteur qui est modifiée. Le résultat typique de la figure 10 montre que le taux de vide est insensible à ce réglage (écarts $<0,6 \%$ de vide) tant que $V L$ eff reste inférieur à l'amplitude déclarée (en fait $<V L O+0,5 V B$ ). Entre $V L O+0,5 V B$ et $V L O+(0,5+C S 1)^{*} V B$, les fluctuations du bruit sont perçues par le système comme du gaz, ce qui conduit à des taux de vide voisins de $100 \%$. Au-delà, le système ne détecte plus rien. Ainsi, la bonne compatibilité entre réglage des signaux et valeurs déclarées au logiciel est aisée à assurer.

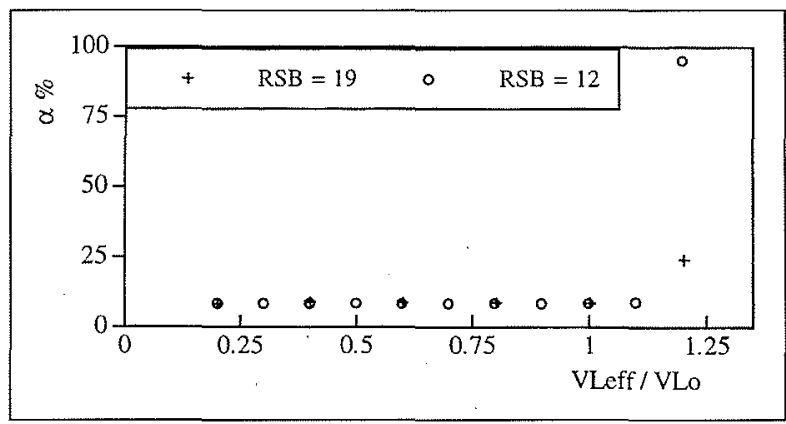

10. Evolution du taux de vide avec le niveau réglé $V L$ eff (sonde conique perpendiculaire à l'écoulement).

La reproductibilité des mesures, testée expérimentalement sur diverses sondes plongées dans des écoulements eau-air, montre que le taux de vide est stable à mieux que $0,2 \%$ de vide et que les variations du temps de présence moyen dans la phase gaz sont limitées à $\pm 2 \%$. Quant à la sensibilité des mesures aux paramètres du tableau 1 , elle reste inférieure aux défauts de reproductibilité. Notons que des valeurs excessives de CS2 (>10) éliminent les bulles les plus petites, et biaisent les mesures de vitesse et de corde (variation $<4 \%$ lorsque CS2 évolue entre 2 et 20 ).

Le système délivre donc des mesures indépendantes de l'intervention de l'opérateur pourvu que les recommandations émises sur le réglage des signaux soient respectées. Cette bonne fiabilité, absente de la plupart des procédures de traitement $[3,2]$, permettra notamment de conduire les qualifications sur des bases solides et de traduire les incertitudes exclusivement en terme de performance de sonde.

\subsection{Qualifications}

Les qualifications ont été réalisées selon une procédure classique, à savoir par intégration de profils de variables locales et comparaison avec des mesures globales.

\subsubsection{Installation expérimentale}

L'installation utilisée consiste en une conduite cylindrique verticale de diamètre intérieur $5 \mathrm{~cm}$, dans laquelle sont produits des écoulements ascendants co-courants eau-air permanents. Deux vannes rapides (temps de fermeture $0,1 \mathrm{sec}$.) situées à $2 \mathrm{~m}$ et $4,5 \mathrm{~m}$ de l'injection permettent d'accéder à la fraction volumique de vide $R G 3$. L'incertitude sur $R G 3$ est de $0,5 \%$ de vide pour un taux de vide supérieur à $6 \%$. En deçà, l'incertitude n'a pas pu être correctement quantifiée, la reproductibilité étant mauvaise (variations $\approx 25 \%$ ). Les débits de liquide et de gaz sont mesurés par des rotamètres étalonnés. La granulométrie des bulles peut être modifiée par changement du système d'injection : pour les essais présentés, la taille typique des bulles est comprise entre 3 et $6 \mathrm{~mm}$. Les sondes sont positionnées à $3,4 \mathrm{~m}$ en aval de l'injecteur. Chaque profi] comporte une trentaine de points de mesure obtenus sur une durée de $5 \mathrm{~min}$, et avec des critères de traitement identiques.

\subsubsection{Incertitude sur le taux de vide}

Deux fractions surfaciques de vide $R G 2 d$ et $R G 2 g$ sont calculées par intégration respectivement des demi-profils droit et gauche symétrisés sur la section. L'écart relatif ( $R G 2-R G 3) / R G 3$, déduit de leur moyenne $R G 2$, est représenté figure 11 pour une sonde conique perpendiculaire à l'écoulement moyen et pour une sonde étirée (OptoflowPhotonetics) parallèle à l'écoulement moyen, et ce pour plusieurs vitesses superficielles $J L$ du liquide. Les barres 
d'erreur verticales correspondent aux valeurs $R G 2 d$ et $R G 2 \mathrm{~g}$ : leur ampleur quantifie la dissymétrie du profil.

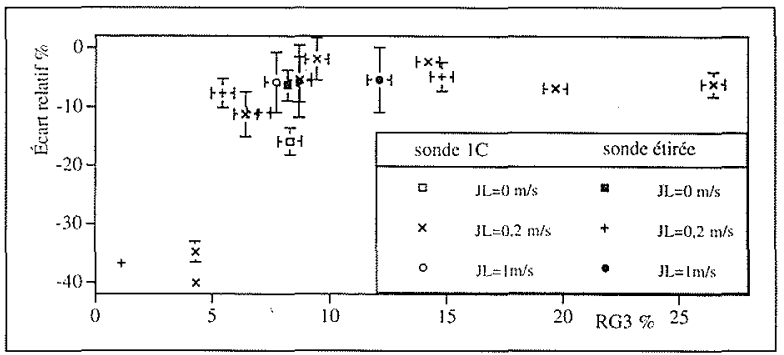

11. Ecart relatif sur la fraction de vide mesurée par sonde optique et par vannes rapides.

Pour une fraction volumique de vide supérieure à $5 \%$, l'écart entre les deux techniques reste inférieur à $11 \%$ pour les deux sondes, excepté à $I L$ nul pour lequel l'erreur atteint $16 \%$. Il est remarquable que les sondes sousestiment systématiquement la fraction de gaz: cette mise en évidence du phénomène d'évitement a été facilité par la qualité du traitement. A faible fraction volumique $(<5 \%)$, les écarts deviennent importants $(35 \%)$. Toutefois, il est difficile de conclure sur l'erreur commise car la mesure par vannes rapides ne constitue plus une bonne référence; nous en voulons pour preuve l'apparition de valeurs de $R G 3$ aberrantes (supérieures à la fraction de débit volumique du gaz) dans cette zone.

\subsubsection{Incertitude sur la vitesse moyenne du gaz}

L'intégrale sur la section de la vitesse moyenne de la phase gaz $\mathrm{Vg}$ pondérée par le taux de présence local de cette phase est comparée au débit volumique global $Q G$. L'écart relatif $(Q g$ mesuré- $Q G) / Q G$ est donné figure $/ 2$ pour une sonde Optoflow parallèle à la vitesse débitante $\left(L^{*} \approx 70 \mu \mathrm{m}\right)$.

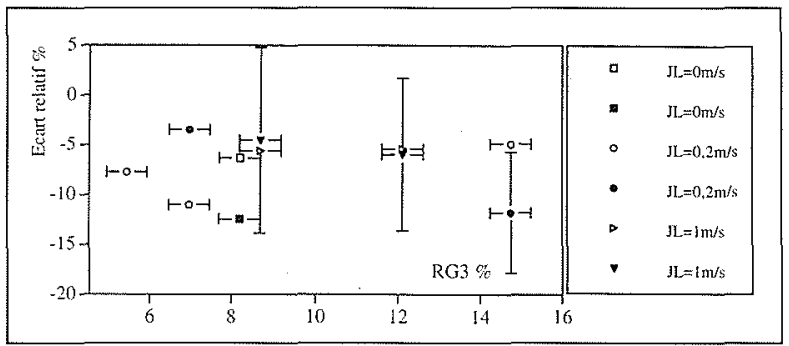

12. Erreurs relatives sur le débit gaz (caractères fermés) et sur la fraction de vide (caractères vides).

L'écart est au plus de l'ordre de $13 \%$ : il est équivalent à l'erreur sur la seule fraction de vide ce qui illustre la bonne précision de l'estimation de la vitesse. Une mesure réalisée à $R G 3 \approx 1 \%$ (non représentée) a foumi un écart sur le débit de $-36 \%$, valeur là encore du même ordre que celle obtenue pour le seul taux de vide. Le test en débit étant indépendant des vannes rapides, la forte sousestimation de a semble donc confirmée. Ceci peut s'expliquer par un évitement plus important lorsque les bulles sont peu nombreuses, évitement qui semble plutôt lié au support de sonde (diamètre $3 \mathrm{~mm}$ ) qu'à la fibre elle-même.

\section{BI-SONDES}

L'originalité des bi-sondes provenant de leur aptitude à mesurer une vitesse de la phase gaz par temps de vol entre pointes sensibles, c'est cet aspect qui retiendra notre attention.

\subsection{Qualification en interfaces contrôlés}

Pour contrôler le bon fonctionnement des bi-sondes, des tests de perçage d'interfaces planes ont été réalisés pour plusieurs orientations du capteur (écartement entre têtes $=1,08 \mathrm{~mm}$ ), et jusqu'à des vitesses de $7 \mathrm{~m} / \mathrm{s}$. Il est apparu que, pour assurer une erreur minimale sur la vitesse, le décalage temporel doit être mesuré à partir des démarrages $T A$ des événements. L'erreur commise évolue alors entre $\pm 25 \%$ pour une sonde orientée selon la vitesse; elle croît fortement en inclinant la sonde. Ces erreurs sont dues à la perturbation de l'interface induite par la sonde amont. Au-delà de $3 \mathrm{~m} / \mathrm{s}$, l'erreur garde un signe constant, correspondant à une sous-estimation de la vitesse de l'interface. Dans ce cas, la flexion des fibres contribue aux défauts de détection.

\subsection{Traitement de signal temps réel, et sensibilité}

Après analyse des techniques de traitement existantes, nous avons retenu le principe de l'association d'évènements dans le domaine temporel. Le traitement temps réel décrit ci-dessus pour la détection des dates $T A$ et $T B$, a donc été appliqué à chacune des voies $\left(f e_{\max }=500 \mathrm{kHz}\right)$. Pour apparier les événements détectés, le post-traitement compare les temps de présence dans la phase gaz : le temps de transit est validé si les $T G$ sont identiques à un pourcentage près imposé. La sensibilité à ce critère est de l'ordre de $10 \%$ sur la vitesse moyenne, et de $20 \%$ sur la corde moyenne. Cette forte sensibilité est d'autant plus préjudiciable à la qualité de la mesure qu'aucune règle ne permet d'en fixer une valeur physiquement pertinente. En outre, un tel critère ne permet pas d'éliminer les associations d'événements incorrectes, dont la probabilité d'occurrence croît lorsque les bulles deviennent plus petites que l'écartement entre sondes.

\subsection{Qualification}

Des qualifications ont été conduites en taux de vide et vitesse sur l'installation décrite au \$2.4.I. Pour la sonde amont, les écarts entre $R G 2$ et $R G 3$ sont du même ordre que ceux présentés figure $1 /$, alors que la fraction de vide mesurée par la sonde aval est systématiquement plus faible de celle issue de la sonde amont. (par exemple, pour $J L=0,2 \mathrm{~m} / \mathrm{s}$, l'erreur augmente de $8 \%$ environ). 
L'écart entre le débit de gaz calculé et le débit de référence $Q G$, illustré figure 13 , atteint des valeurs élevées $(30 \%)$. Il est remarquable que la majorité des essais conduisent à une sur-estimation du débit. Le taux de vide étant assez correctement prédit, l'erreur est donc imputable à la mesure de vitesse. L'augmentation sensible de la sur-estimation de $V g$ avec $R G 3$ s'explique notamment par l'accroissement des associations illicites entre événements avec le nombre de bulles présentes dans l'écoulement.

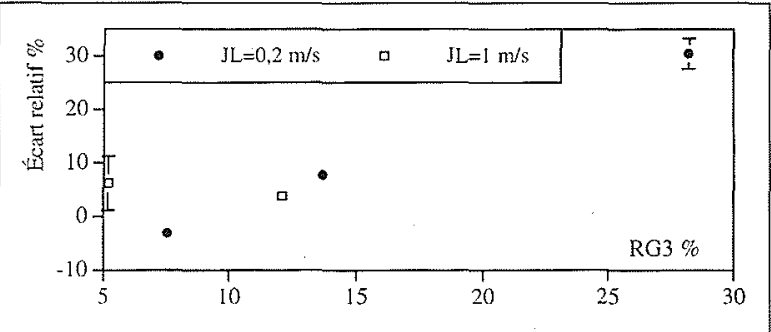

13. Ecart relatif sur le débit gaz mesuré par bi-sonde pour un critère d'association de $5 \%$.

Ainsi, l'existence d'un degré de liberté dans le traitement, et la multiplicité des situations conduisant à des estimations de temps de vol erronées, rendent l'utilisation des bi-sondes plus délicate que les mono-sondes. Ces dernières, complétées par un traitement adapté, ont démontré leur capacité à caractériser les écoulements eau-air; et ce de manière fiable.

\section{PERSPECTIVES}

Les travaux présentés dans cet article se poursuivent en vue de compléter les essais de perçage (effet de $\gamma$, sondes 3C à longueur de cylindre réduite, contamination) ainsi que les qualifications (sonde $3 \mathrm{C}$, influence de la granulométrie des bulles, écoulements complexes à forte vitesse de la DEREDF) : l'ensemble des résultats fera l'objet de la thèse d'E. Barrau [9]. Par ailleurs, les chaînes de mesure réalisées pour les mono-sondes comme pour les bi-sondes ayant prouvé leur fiabilité, leur valorisation a été engagée par EDF et le CNRS.
La bonne maîtrise des géométries de sondes étant assurée, il est maintenant possible de revenir de façon approfondie sur les aspects hydrodynamiques. Pour cela, le LEGI a démarré des études sur l'influence de la nature do fluide, et sur l'évolution du film liquide lors d'interactions sondes/gouttes, ce dernier thème étant lié à l'application de sondes mono-fibre à des écoulements à gouttelettes.

Reste posé le problème d'une qualification sûre à faible taux de vide, qui devrait pouvoir être résolu par les systèmes de mesure développés par J. Leblond dans le cadre du GREDIC. Enfin, l'extension à la détection du sens de la vitesse parait désormais accessible grâce aux géométries conique-cylindro-coniques proposées.

\section{Références}

[1] JONES O., DelhaYe J.M. (1976). - Transient and statistical measurement techniques for two-phase flows: a critical review, Int. J. Multiphase Flow, vol. 3.

[2] CarTel liler A., AChard J.L. (1991), - Local phase detection probes in fluidffluid wo-phase flows, Review of Scientific Instruments, vol. 62, $n^{\circ} 2$.

[3] CARTELLIER A. (1990). - Oprical probes for void fraction measurements: characterization of performances, Review of Scientific Instruments, vol. $61,1^{\circ} 2$

[4] CARTEI I.1ER A. (1992). - Simultaneous void fraction measurement, bubble velocity, and size estimate using a single oprical probe in gas-liquid no-phase flows, Review of Scientific Instruments, vol. $63, n^{\circ} 11$

[5] CARTEllier A. (1991). - Contral EDF n' T37L01 2.J7100/RNE 292 - CNRS n 509687 intitulé : Conceprion el réalisation d'un logiciel de traitement des signaux déliwés par les sondes opriques monofibre pour les écoulements diphasiques gaz-liquide, Rapports 1 à 4 .

16] Abuaf N., Jones C., Zimmer G., Leonhardt W., Saha P. (1978). - BNL flashing experiments : test facility and measurement techmiques, 2nd CSNI Specialists Meeting, June 1214, Paris

17) CL.ARK N. TURTON R. (1988). - Chord length distributions related to bubble size distributions in multiphase flows, Int J. Multiphase Flow, vol. 14

[8] Machane R., Canot E. (1995). - Emergence de corps à la surface libre d'un fluide non visquenx, $12^{\circ}$ Cong. Français de Mécanique, 4-8 Sept., Strasbourg.

[9] Barrau E. Cartellier A. (1993-1995). - Contrat EdF $n^{\circ}$ T34L01-2K6727/RNE 402 - CNRS n ${ }^{\circ}$ 720744/00 intitulé : Trairemem en remps réel pout sondes optiques: conception et validation, Rapports 1 à 9 